\title{
CULTURE AND COMMUNiCATION IN THE ENGINEERING ClassRoom
}

\author{
Kathleen Clarke \\ IEEQ Program, Faculty of Engineering \\ University of Manitoba, Winnipeg, MB Canada \\ $<$ Kathleen_Clarke@umanitoba.ca $>$
}

\begin{abstract}
This session will present successful strategies for instructors and internationally-educated engineering graduates (IEGs) to manage issues of culture and communication in the engineering classroom with a focus on aspects of the innovative Internationally Educated Engineers Qualifications (IEEQ) Program at the University of Manitoba. The session will also be of interest to those working with international students and mature/adult learners.
\end{abstract}

Several factors influence the successful integration of IEGs into Canadian engineering careers including, but not limited to achieving professional registration and navigating professional, cultural and communication differences. Many IEGs in Manitoba opt to take engineering courses at the University of Manitoba to fulfill the academic requirements for professional registration by enrolling as "special students not seeking degree", or by completing the IEEQ Program. The classroom itself presents many challenges for IEGs in terms of differences in education systems and academic processes, and like the workplace, the classroom can also be replete with cultural and communication differences, and differences in professional practice. These complex challenges can be time consuming and costly for all parties. Kathleen Clarke will provide a helpful framework of effective practices and lessons learned from the ongoing IEEQ experience in Manitoba. 